is now causing the strong torque. A similar sequence of events could have occurred in comet $103 \mathrm{P} /$ Hartley 2, which was visited by the Deep Impact Extended Investigation (DIXI) space mission ${ }^{8,10}$ in 2010.

Extrapolating comet 41P's rotation rate forward in time, Bodewits et al. predict that the period would have exceeded 100 hours in mid-2017. Such an extremely slow rotation would no longer stabilize the comet's spatial orientation, so that even small torques could make it wobble like a spinning top. If the current strong torque persists, it might eventually drive the comet to spin up again, possibly about a different axis.
A change in comet 41P's rotation axis would affect the seasonal distribution of heating across the body's surface, the associated levels of activity and the pattern of mass transport between different regions ${ }^{11}$. The global process of cometary erosion might therefore be redirected. Observations from the end of the 2017 activity period and from the next perihelion passage in 2022 could document this yetto-be-seen phase of cometary evolution, and reveal valuable information about the nature of comets and other planetary bodies.

Jessica Agarwal is at the Max Planck Institute for Solar System Research,
37077 Göttingen, Germany.

e-mail:agarwal@mps.mpg.de

1. Whipple, F. L. Astrophys. J. 111, 375-394 (1950).

2. Bodewits, D., Farnham, T. L., Kelley, M. S. P. \& Knight, M. M. Nature 553, 186-188 (2018).

3. Rubincam, D. P. Icarus $148,2-11$ (2000)

4. Marsden, B. G. Astron. J. 74, 720-734 (1969)

5. Keller, H. U., Mottola, S., Skorov, Y. \& Jorda, L. Astron. Astrophys. 579, L5 (2015).

6. Hirabayashi, M. et al. Nature 534, 352-355 (2016).

7. Jewitt, D. et al. Astrophys. J. 829, L8 (2016).

8. Steckloff, J. K., Graves, K., Hirabayashi, M., Melosh, H. J. \& Richardson, J. E. Icarus 272, 60-69 (2016).

9. Combi, M. SOHO SWAN Derived Cometary Water Production Rates Collection (NASA, 2017).

10. A'Hearn, M. F. et al. Science 332, 1396-1400 (2011)

11. Keller, H. U. et al. Mon. Not. R. Astron. Soc. 469, S357-S371 (2017).

\title{
Neuronal plasticity in nematode worms
}

\section{Neuronal activity induces changes in the connectivity of a neuron called DVB in adult male nematode worms. This discovery provides an opportunity to study a fundamental process in this powerful model organism. SEE ARTICLE P.165}

\section{SCOTT W. EMMONS}

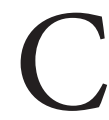
entral to the function of the nervous system is its dynamic ability to undergo changes, for instance in the physiological properties of its constituent neurons, the synaptic connections between them, and the characteristics of individual synapses. The hypothesis that neuronal activity can lead to such plasticity, first proposed by the neurophysiologist Donald Hebb in 1949, is fundamental to brain science, and has been confirmed in many studies ${ }^{1}$. On page 165 , Hart and Hobert ${ }^{2}$ describe an example of experience-dependent neural plasticity in the nematode worm Caenorhabditis elegans, a species in which this phenomenon has been little studied ${ }^{3}$.

It is important to demonstrate this already well-described and widely studied neural phenomenon in a nematode because $C$. elegans is not just any worm, but a powerful experimental model. Genetic studies in C. elegans have led to the discovery of several molecular components common to all nervous systems. Furthermore, a complete map of neural connectivity in the nematode nervous system has been available for more than 30 years ${ }^{4,5}$ - such a connectome is not yet available for any other animal.

Assembly of the C. elegans connectome was made possible not only by the worm's tiny size (1 millimetre long), but also because its cells are constant in number and identity, and its synaptic connections are largely conserved between individuals. These properties, together with the fact that connectivity data were obtained from only a few individuals, have created the impression that the $C$. elegans nervous system is exceptional in having a rigid and constant structure. Intuition suggests that this cannot be the case - the worm's nervous system is so complex that it must be based on dynamic mechanisms. But few examples of variability in C. elegans neurons have been described until now.

The C. elegans inhibitory neuron DVB makes different connections in the worm's two sexes: males and hermaphrodites ${ }^{4,6}$. A single process extends towards the head of the worm in both sexes, and a male-specific outgrowth towards the tail leads to the formation of synaptic connections to a neuron and muscles that control the movement of the male's spicules - a pair of hardened structures that insert into the vulva of the hermaphrodite during mating ${ }^{6}$ (Fig. 1). The formation of these new synapses, and the loss of some old ones, mean that spicule movement comes under the

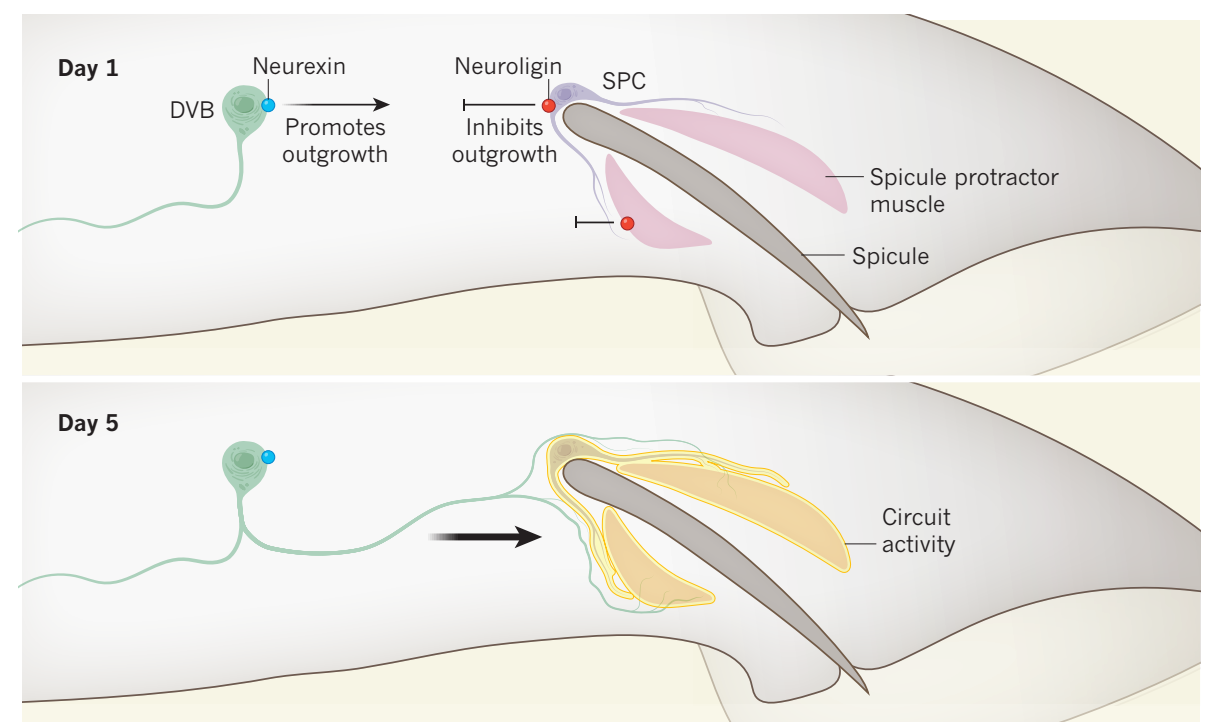

Figure 1 | Activity-dependent neuronal outgrowth in nematodes. Hart and Hobert ${ }^{2}$ examined the neuron DVB in nematode worms (Caenorhabditis elegans). They report that, between days one and five of adulthood in male worms, DVB grows towards, and makes synaptic connections onto, spicule protractor muscles and the spicule neuron SPC, which control a male-specific mating behaviour involving movement of a structure called the spicule. This outgrowth is regulated, at least in part, by two celladhesion proteins: neurexin is expressed by DVB and promotes outgrowth; and neuroligin is expressed by the spicule protractor muscles and SPC, and inhibits outgrowth. The authors show that the expression of neuroligin is repressed when the male undergoes copulatory behaviours, activating these muscles and SPC - DVB outgrowth is therefore activity dependent. 
inhibitory control of DVB. This refinement improves the male's mating efficiency ${ }^{7}$. Hart and Hobert now show that this male-specific outgrowth of DVB occurs between days 1 and 5 of adult life. The outgrowth produces a branching neuronal architecture that, unlike many neuronal circuits in C. elegans, varies between individuals.

Hart and Hobert used fluorescent 'reporter' proteins to visualize DVB outgrowth and synapse formation. Their analysis reveals that outgrowth does not occur if the male does not experience copulatory activity. The authors then mimicked natural behaviours by using sophisticated genetic techniques to activate or inhibit the signalling or movement of DVB's target neuron and muscles, respectively. This shows that activity in DVB's targets stimulates the neuron's outgrowth.

What molecular pathways might mediate DVB outgrowth? Neural cell-adhesion proteins are expressed on cell surfaces in the nervous system. They have extracellular protein-protein interaction domains that can mediate communication between cells, and are thought to have a role in encoding and building the nervous system's synaptic structure ${ }^{8}$. Two of the best-studied proteins in this class are neurexin and neuroligin, which can interact with one another and are involved in synapse formation and regulation ${ }^{9}$. As such, they were natural candidates for Hart and Hobert to test.

The authors examined the roles of these proteins by combining genetic deletion or overexpression of the proteins with stimulation or suppression of activity in the circuit. These analyses led to several findings. First, neurexin is expressed in DVB and is required for DVB outgrowth. Second, the activity of neurexin is inhibited by neuroligin, which is expressed in male sex circuits and muscles. Third, neuroligin expression is suppressed by activity in the circuit, which explains why DVB outgrowth is activity dependent. Precisely how neuroligin inhibits DVB outgrowth, and whether the two proteins physically interact in this setting, remain to be determined.

Hart and Hobert's work brings together three areas of study in neuroscience: outgrowth, branching and target selection in plastic neurons; control of these processes through neuronal activity; and the function of neural cell-adhesion proteins. The value of the study therefore lies not only in the discovery of a new phenomenon, but also in the framework it provides for making more discoveries.

Analysis of C. elegans mutants will make it possible to identify additional molecules that affect DVB outgrowth, such as the binding partner of neurexin that stimulates outgrowth. The intracellular mechanisms that drive DVB outgrowth, and how they are controlled by interactions between neurexin and its binding partner, can then be analysed. Other questions for study include how DVB knows where to send processes, how its axonal extensions recognize appropriate synaptic targets, and precisely how circuit activity controls neuroligin expression.

Finally, Hart and Hobert found that these events occur only in males. The authors attempted to stimulate DVB outgrowth in hermaphrodites, but their results suggest that neither circuit activity nor the neurexinneuroligin pathway are by themselves sufficient to do this. Other work ${ }^{10}$ in C. elegans suggests that it is the complement of sex chromosomes (two X chromosomes in the hermaphrodite and only one in the male) in the cells of the circuit that ultimately makes them respond to sex-neutral pathways in sex-appropriate ways.

Genetic studies ${ }^{9}$ have implicated mutations in neural cell-adhesion genes, including neurexin and neuroligin, as the bases of psychiatric disorders, partly because of the roles of these genes in neural plasticity. Progress in unravelling details of the molecular pathways underlying their activity could therefore have profound implications for understanding not only learning and memory, but also mental disorders and their sex-specific expression.

Scott W. Emmons is in the Department of Genetics and the Dominick P. Purpura Department of Neuroscience, Albert Einstein College of Medicine, New York,

New York 10461, USA.

e-mail:scott.emmons@einstein.yu.edu

1. Holtmaat, A. \& Svoboda, K. Nature Rev. Neurosci. 10, 647-658 (2009).

2. Hart, M. P. \& Hobert, O. Nature $553,165-170$ (2018).

3. Jin, Y. \& Qi, Y. B. Curr. Opin. Neurobiol. 48, 97-105 (2017).

4. White, J. G., Southgate, E., Thomson, J. N. \& Brenner, S. Phil. Trans. R. Soc. B 314, 1-340 (1986).

5. Emmons, S. W. Phil. Trans. R. Soc. B 370, 20140309 (2015).

6. Jarrell, T. A. et al. Science 337, 437-444 (2012).

7. LeBoeuf, B. \& Garcia, L. R. G3 Genes Genomes Genet. 7, 647-662 (2017)

8. Williams, M. E., de Wit, J. \& Ghosh, A. Neuron 68, 9-18 (2010).

9. Südhof, T. C. Cell 171, 745-769 (2017).

10.Portman, D. S. J. Neurosci. Res. 95, 527-538 (2017).

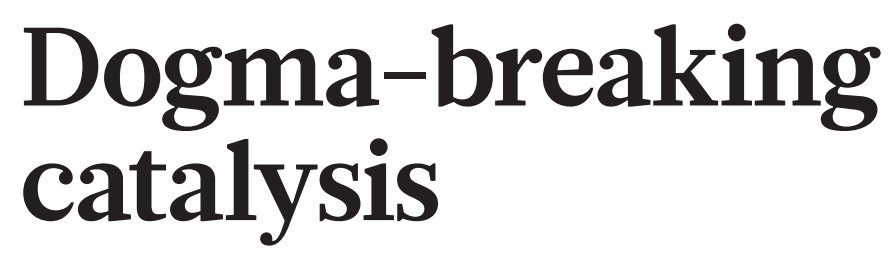

\begin{abstract}
The catalysts conventionally used for industrially important hydrogenation reactions are expensive and generate toxic residues. Catalysts have now been reported that might lead to cheaper, less toxic alternatives.
\end{abstract}

\section{DOUGLAS W. STEPHAN}

$\mathrm{R}$ eactions of hydrogen gas with organic compounds are performed on a large scale worldwide by the chemicals industry ${ }^{1}$. Such hydrogenation reactions are essential to the production of numerous commercial goods, including many polymers, foodstuffs and pharmaceuticals. However, a catalyst is needed to provide a thermodynamically accessible reaction pathway that allows hydrogenations to occur. Until the past decade or so, it was thought that these catalysts must derive from transition metals, but there is now a growing list of alternatives. Writing in Nature Catalysis, Bauer et al. ${ }^{2}$ add to that list by reporting effective hydrogenation catalysts derived from alkaline-earth elements - the group of metals that includes calcium.

About 100 years ago, the chemist Paul Sabatier was the first to recognize that amorphous metals could act as catalysts to mediate the hydrogenation of organic substrates ${ }^{3}$. By the middle of the twentieth century, the emergence of the subdiscipline of organometallic chemistry led to the development of a wide variety of transition-metal complexes that are highly effective catalysts for these reactions ${ }^{4}$. Soluble transition-metal catalysts have undergone continual development to offer higher and higher reactivities. In addition, judicious changes to the ligand molecules bound to the metal atom were found to control the reactivity and selectivity of the catalytic complexes - not only the substrate selectivity, but also the stereoselectivity (the $3 \mathrm{D}$ geometric arrangement of atoms generated in the product). Despite these advances, most catalysts used in industrial processes are derived from the metals platinum, palladium, rhodium and ruthenium, which are expensive, toxic and rare.

The cost of the precious metals in such catalysts is not the only expense associated with their use - the removal of toxic catalyst residues from the products is also costly. This, together with increasing environmental concerns, has prompted efforts to find alternatives to conventional hydrogenation catalysts. One strategy that uses the principles of 